\title{
Standounkt
}

Unternehmenskommunikation und Arbeitskultur

\section{Das Dilemma mit der Glaubwürdigkeit}

Die Verantwortung von Unternehmen spielt eine immer stärkere Rolle. Wenn Betriebe Verantwortung für Andere oder die Umwelt demonstrieren, wird dies in der Regel nur dann honoriert, wenn das Unternehmen eine gewisse Glaubwürdigkeit besitzt. Doch Glaubwürdigkeit aufzubauen ist ein langwieriger Prozess, und sie ist vergleichsweise schnell wieder zu verlieren. Von Bernd Lorenz Walter

n Zeiten der Globalisierung und insbesondere im Rahmen der derzeitigen Finanzkrise sehen sich Unternehmen einer schwindenden Glaubwürdigkeit ausgesetzt.

So betonte Bundespräsident Horst Köhler in seiner Rede zur Verleihung des Max-Weber-Preises für Wirtschaftsethik am 27. Mai 2008 in Berlin, dass 42 Prozent der Bundesbürger die Einschätzung teilen, dass die meisten Wirtschaftsführer korrupt sind. Nicht einmal jeder fünfte Bundesbürger findet, dass die soziale Marktwirtschaft gut funktioniert.

\section{Gewinnstreben und gesell- schaftliche Verantwortung}

Schwierig scheint vor diesem Hintergrund für Unternehmen, eine Verknüpfung zwischen der Übernahme gesellschaftlicher Verantwortung und ökonomischen Notwendigkeiten herzustellen. Das Gewinnstreben, das Unternehmen per se von anderen Organisationsformen unterscheidet, wird mit nimmer satter Raffgier gleichgesetzt.

Wenn das Unternehmen von seinem vermeintlichen Reichtum etwas für wohltätige Zwecke abgibt, wird das eher als Hohn verstanden und weniger als Zeichen sozialer Verantwortung. Denn für die meisten ist die Wirtschaft eine Black-Box oder es sind „Die-da-oben“, die mit den anderen ohnehin machen, was sie wollen. Man traut den Unternehmen einfach nicht, wenn sie sich selbst und ihresgleichen wohlmeinend auf die Schultern klopfen.
Die Grundvoraussetzung für die Übernahme gesellschaftlicher Verantwortung ist daher zunächst die Glaubwürdigkeit. Ohne sie verpuffen die durchaus redlich gemeinten Bemühungen, Verantwortung zu demonstrieren. Damit haben vor allem die Energiekonzerne zu kämpfen. Sie verkörpern nach wie vor die Abzocker der $\mathrm{Na}$ tion, auch wenn sie noch so sehr betonen, welchen gesellschaftlichen Beitrag sie eigentlich leisten und wie viel sie in erneuerbare Energie oder Energieeffizienz investieren. Auch die Pharmaindustrie leidet unter der mangelnden Glaubwürdigkeit, obwohl sie grundsätzlich Produkte herstellt, die dem gesellschaftlichen Wohl dienen. Trotzdem wird die Branche nach wie vor als Teil des Problems und nicht als Teil der Lösung wahrgenommen.

\section{Verantwortung als innere Einstellung}

Wichtig ist zu erkennen, dass Verantwortung zunächst einmal etwas mit einer inneren Einstellung zu tun hat. Es geht nicht um ein Sowohl-als-auch, sondern um einen ethisch und moralisch geprägten Bewusstseinsprozess, der sich wie ein roter Faden durch das Handeln des Unternehmens zieht. In diesem Sinne ist es nicht maßgeblich, wie der Profit sozial und ökologisch verwendet werden kann, sondern auf welche Art und Weise dieser erwirtschaftet wird. Bereits die Startseiten im Internet vieler Unternehmen suggerieren das Gegenteil: hier das Business und dort die Verantwortung.
Glaubwürdige Verantwortung ist identitätsstiftender Teil einer wertebasierten Unternehmenskultur (value-driven). Sie wirkt entsprechend nur von innen nach außen und setzt daher das Evaluieren einer Analyse des Verantwortungsprofils voraus.

\section{Wege aus dem Teufelskreis}

Daraus lassen sich konkrete Ansatzpunkte für eine performanceorientierte Konzeptentwicklung des Verantwortungsprofils ableiten. Das Ergebnis dieses Prozesses ist wiederum Bedingung für weitere Maßnahmen wie das Entwickeln von Leitlinien, Kodizes oder Selbstverpflichtungen.

Die Evaluation des Verantwortungsprofils muss aber in einem weiteren Schritt mit einer Outside-in-Perspektive abgeglichen werden. Diese beinhaltet im Wesentlichen das Monitoring der Erwartungshaltungen der Stakeholder und der gesellschaftsrelevanten Fragestellungen. Dieses Monitoring darf sich nicht nur auf ein systematisches Beobachten beschränken, sondern muss vielmehr dialogorientiert ausgerichtet werden. Auch in der anschließenden Durchführung diverser Programme zur gezielten Förderung und Demonstration des Verantwortungsbewusstseins ist ein Dialog erfolgskritisch.

Glaubwürdige Verantwortung setzt zu allererst ein klares Bekenntnis der Unternehmensspitze voraus. Ihr Führungsverhalten und ihre charakterliche Integrität ist die unternehmenseigene Benchmark für Glaubwürdigkeit bei der Übernahme von Verantwortung.

I AUTOR + KONTAKT

Bernd Lorenz Walter arbeitet als Berater für strategische Kommunikation und Reputation in Berlin. Bernd Lorenz Walter, Katzbachstr. 3, 10965 Berlin. Tel.: +4930443561 22/23, E-Mail:welcome@BLWalter.com 
(c) 20I0 Authors; licensee IÖW and oekom verlag. This is an article distributed under the terms of the Creative Commons Attribution Non-Commercial No Derivates License (http://creativecommons.org/licenses/by-nc-nd/3.o/), which permits unrestricted use, distribution, and reproduction in any medium, provided the original work is properly cited. 